\title{
CARACTERIZAÇÃO DAS DESIGUALDADES EDUCACIONAIS COM DADOS PÚBLICOS: DESAFIOS PARA CONCEITUAÇÃO E OPERACIONALIZAÇÃO EMPÍRICA
}

Maria Teresa Gonzaga Alves

Professora associada do Departamento de Ciências Aplicadas à Educação da Universidade Federal de Minas Gerais (UFMG). Belo Horizonte,MG, Brasil. E-mail: mtga@ufmg.br

Orcid: 0000-0001-5820-4311

http://dx.doi.org/10.1590/0102-189214/110

\section{Introdução}

As bases da abordagem sociológica no estudo da educação formal são encontradas em autores clássicos, como Émile Durkheim e Max Weber, mas somente a partir do fim da década de 1950 a sociologia da educação se institucionalizou como um campo de pesquisa. Um fator primordial para isso foi a disponibilidade de dados em larga escala produzidos em pesquisas demográficas e grandes surveys educacionais realizados em países desenvolvidos (Forquin, 1995). Esses levantamentos de dados tiveram como motivação a investigação das desigualdades de acesso e de resultados escolares entre classes sociais, grupos raciais, gênero local de moradia e outras variáveis adscritivas, assim como as condições da oferta educativa (perfil dos professores, infraestrutura, recursos educacionais). Esses temas assumiram posição central nas políticas de igualdade de oportunidades do pós-guerra, 
por exemplo nos Estados Unidos, a partir da Lei de Direitos Civis que pressupunha grandes desigualdades entre as escolas da nação (Brooke e Soares, 2008; Karabel e Halsey, 1977).

Nos Estados Unidos, o conhecido Relatório Coleman foi fundamental para a compreensão da associação entre origem social, étnico-racial, regional e outras variáveis estruturais na explicação das desigualdades em resultados escolares (Coleman et al., 1966). Na França, sociólogos, com bases em dados demográficos longitudinais, conduziram análises sobre as desigualdades de trajetórias educacionais no sistema de ensino e a constituição do paradigma da reprodução - o conjunto de teorias fundamentais no campo da sociologia da educação para a análise das desigualdades educacionais - seria simplesmente impensável sem os fatos estatísticos estabelecidos nesses anos (Nogueira, 1990).

A partir do fim dos anos 1970, um conjunto de publicações de países desenvolvidos passou a disputar as conclusões 190 do Relatório Coleman e outros levantamentos do tipo a respeito do peso da origem social na educação. $\mathrm{O}$ argumento central dos autores era de que a variação entre as escolas não poderia ser negligenciada na explicação dos resultados escolares (Brookover et al., 1979; Rutter et al., 1979). Essas pesquisas configuraram um novo subcampo de estudo, chamado de sociologia dos estabelecimentos escolares, ou pesquisa sobre efeito escola, ou, ainda, pesquisa em eficácia escolar (Brooke e Soares, 2008; Mafra, 2003). Nessa nova vertente, os autores argumentavam que era preciso abrir a caixa-preta da escola, dirigir o foco para o contexto escolar e os processos que ocorriam dentro dos estabelecimentos de ensino sem, evidentemente, negar a influência de fatores extraescolares, como a origem social dos estudantes e os investimentos em educação (Alves e Soares, 2007). Essas pesquisas também utilizam dados quantitativos, mas estudos com abordagens qualitativas também prosperaram, muitas vezes conjugando os dois métodos. 
Vale destacar que as pesquisas empíricas no campo da Sociologia tiveram grande impulso com o desenvolvimento computacional verificado na segunda metade do século XX, o que viabilizou a aplicação de modelos estatísticos em grandes bases de dados. Na área da educação, a partir dos anos 1990 o desenvolvimento de modelos estatísticos apropriados para análise de dados com estrutura hierárquica - tipicamente alunos agrupados em escolas -, a conhecida modelagem multinível, foi fundamental para pesquisas sobre efeito dos estabelecimentos de ensino (Lee, 2008; Willms, 1992). Em termos analíticos, com esses modelos "começamos a ver as ações dos indivíduos como mediadas pelas ações dos outros com os quais eles estão em contato e pelas instituições às quais eles pertencem" (Goldstein, 2001, p. 96).

No Brasil, não foi diferente. A produção de dados sobre resultados educacionais - rendimento e desempenho escolar - avançou a partir dos anos 1990, após a Constituição de 1988 e a aprovação da Lei de Diretrizes e Bases da Educação Nacional (1996) como evidências de garantia do direito constitucional à educação (Soares, 2016).

Convém mencionar, entretanto, que a tradição da produção de dados estatísticos sobre educação é anterior. Parte importante da pesquisa em sociologia da educação e áreas correlatas - como a demografia e a economia educacional se desenvolveu graças à existência de dados públicos populacionais e escolares sobre a educação formal da população. O Instituto Brasileiro de Geografia e Estatística (IBGE) é responsável pelo Censo Demográfico e Pesquisa Nacional por Amostra de Domicílio (Pnad), que descrevem o acesso à educação formal, a alfabetização e o alcance educacional da população (Ferraro, 2002; Valle Silva e Hasenbalg, 2000; Valle Silva e Souza, 1986).

O Instituto Nacional de Estudos e Pesquisas Educacionais “Anísio Teixeira” (Inep) é responsável pelas pesquisas realizadas nos estabelecimentos escolares, como o Censo da 
Educação Básica, o Censo da Ensino Superior - levantamentos sobre matrículas, pessoal docente e infraestrutura dos estabelecimentos de ensino. Essas pesquisas também produzem os dados do rendimento escolar (aprovação, reprovação e abandono) e o fluxo escolar, que são fundamentais para o acompanhamento da trajetória educacional das coortes que ingressam nos sistemas de ensino (Klein, 2003).

O Inep também é o responsável pelas pesquisas de avaliação educacional de base nacional como as que compõem o Sistema de Avaliação da Educação Básica (Saeb), o Exame Nacional do Ensino Médio (Enem), e o Exame Nacional de Desempenho de Estudantes (Enade). Além disso, o Inep coordena a participação do Brasil em avaliações comparativas internacionais, como o programa de avaliação de estudantes de 15 anos, mais conhecido por sua sigla internacional: PISA, e estudos regionais na América Latina (Alves e Franco, 2008).

192 O IBGE e o Inep produzem dados ricos, sistemáticos e de qualidade que possibilitam investigar as relações entre escolaridade e desigualdades sociais, raciais, mobilidade social ou ocupacional (Valle Silva e Hasenbalg, 2000). As pesquisas sobre efeito escola, assim como no cenário internacional, se desenvolveram posteriormente, trazendo contribuições importantes para a análise do contexto escolar, desigualdades entre escolas e sistemas de ensino e fatores associados aos resultados educacionais (Alves e Franco, 2008).

Neste texto, mostrarei exemplos da potencialidade dos dados produzidos pelo Inep, especificamente os dados da Prova Brasil - avaliação em larga escala de escolas públicas de ensino fundamental que faz parte do Saeb - para o estudo das desigualdades educacionais. $\mathrm{O}$ primeiro estudo caracteriza as desigualdades de resultados escolares das escolas públicas brasileiras segundo grupos sociais. O segundo apresenta evidências sobre as condições da oferta educativa nas escolas públicas e a relação com o efeito das escolas. 


\section{Evolução das desigualdades de aprendizado no ensino fundamental público: hiato entre grupos sociais de 2007 a 2017}

Em uma pesquisa com dados da Prova Brasil de 2005 a 2013, observamos dois fenômenos importantes sobre a evolução do desempenho escolar (Alves, Soares e Xavier, 2016). Primeiro, mostramos que o aprendizado dos alunos das escolas públicas de ensino fundamental melhorou substancialmente nesse período; e segundo, revelamos que apesar do avanço médio, as diferenças entre grupos de alunos discriminados por gênero, raça/cor e nível socioeconômico (NSE) aumentaram. Ao que parece, os investimentos para a melhoria da educação pública, que cresceram no país a partir de 2003 (Cavalcanti e Oliveira, 2019), contribuíram para elevar a média educacional de uma forma geral, mas a melhoria não beneficiou igualmente os grupos sociais.

Os dados analisados nesse trabalho são anteriores ao período de crise política do país, sobretudo após as eleições de 2014, que afetou a economia e a sociedade de forma geral. Isso nos levou a interrogar se a nova conjuntura teria afetado a qualidade da educação. Especificamente, será que as tendências de melhoria do desempenho escolar no ensino fundamental verificadas até 2013 se mantiveram no período seguinte? As desigualdades entre grupos sociais continuaram a crescer ou teriam atenuado? Para responder a essas questões, as análises do artigo foram atualizadas especialmente para esta comunicação, acrescentando os dados mais recentes da Prova Brasil referentes aos anos de 2015 e 2017. Uma análise mais detida da série histórica da Prova Brasil foi realizada por Alves e Ferrão (2019).

Faço aqui um parêntese para os leitores que não conhecem detalhes técnicos dessas avaliações. Podemos comparar a evolução do aprendizado entre edições da Prova Brasil graças à metodologia empregada nesse tipo de avaliação. O aprendizado mensurado na Prova Brasil refere às proficiências dos alunos em leitura e Matemática, estimadas por meio da Teoria da Resposta 
ao Item (TRI) (Hambleton, 1993). A TRI permite a comparação dos resultados obtidos entre as séries avaliadas (quinto e nono ano do ensino fundamental) e entre as edições da Prova Brasil (de 2005 a 2017) e também da Prova Brasil com o Saeb - avaliação que inclui escolas privadas. Em outros termos, a escala de proficiência forma um continuum com valores de 0 a 500 , e tipicamente os alunos avaliados da primeira etapa do ensino fundamental apresentam proficiências médias mais baixas na escala e os alunos da etapa final médias mais altas. Na comparação entre edições da Prova Brasil, se houve aumento da média, isso pode ser atribuído a uma melhora na qualidade do ensino ou do aprendizado, e não ao fato de o teste, por exemplo, ter sido mais fácil. Uma eventual piora nas médias pode ser interpretada como problema na qualidade do ensino/aprendizado e não pelo fato de os testes terem sido mais difíceis.

Neste artigo, apresentamos a evolução das médias dos resultados gerais e por grupos de alunos de 2007 a 2017, ou seja, por 10 anos de Prova Brasil. Nos gráficos 1 e 2 apresentamos os resultados gerais, isto é, as médias das proficiência dos alunos das escolas públicas de ensino fundamental. Observem que as médias do nono ano são mais altas (linha verde) porque, como explicado, a escala é a mesma. Tipicamente os alunos do final do ensino fundamental apresentam médias mais altas no intervalo da escala. Isso é uma evidência do aprendizado acumulado de uma etapa a outra. Nesses gráficos, o que importa é observar as tendências, que são crescentes, tanto em leitura quanto em Matemática, em todo o período. Mas as linhas de tendências para Matemática sempre estão mais suaves e, de 2015 para 2017, houve uma desaceleração do crescimento das médias; as linhas estão menos inclinadas, sobretudo no nono ano.

Os próximos resultados destacam o hiato do aprendizado segundo gênero, raça/cor e NSE dos alunos. Os achados são consistentes com os estudos sociológicos em relação ao peso das variáveis de origem no desempenho dos alunos e confirmam os padrões de desigualdades educacionais conhecidos da literatura. 
Gráfico 1

Evolução do aprendizado em leitura nas

escolas públicas de ensino fundamental: 2007 a 2017

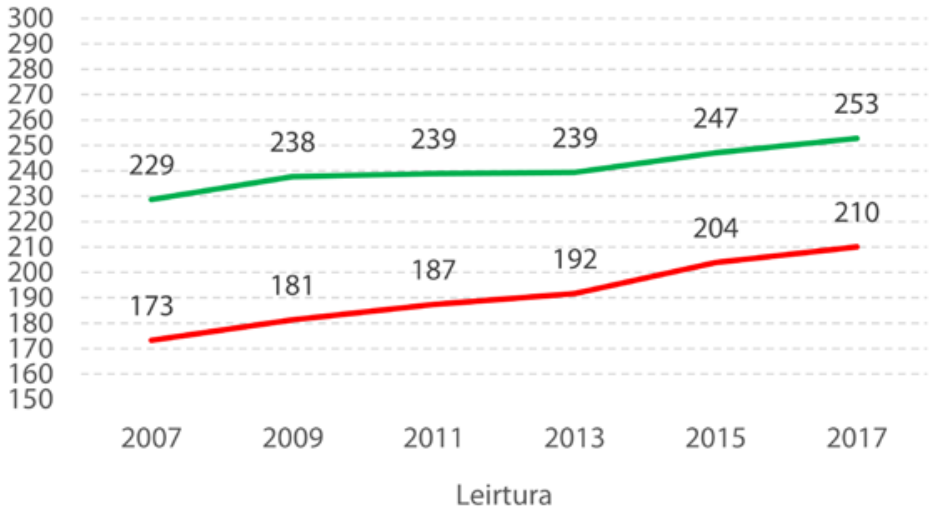

-5o ano - 90 ano

Fonte: Elaboração própria com dados da Prova Brasil/Inep.

Gráfico 2

Evolução do aprendizado em matemática nas

escolas públicas de ensino fundamental: 2007 a 2017

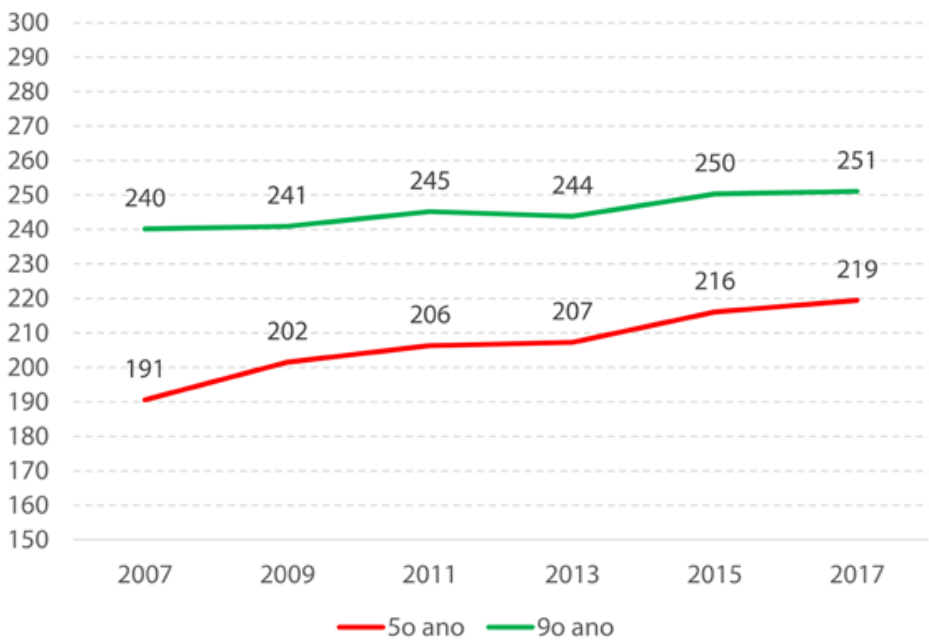

Fonte: Elaboração própria com dados da Prova Brasil/Inep. 
No Gráfico 3 estão as tendências de aprendizado em leitura no quinto ano, segundo a variável sexo; e, no Gráfico 4, o mesmo para o nono ano. Observem que há um hiato persistente entre alunas e alunos desde os anos iniciais do ensino fundamental. Os meninos estão sistematicamente em desvantagem em leitura, a diferença varia de 10 pontos, em 2007, a 12,7 pontos, em 2015. Em Matemática, ocorre o contrário: os meninos têm médias mais altas, mas nesse caso, o hiato cresce muito de uma etapa para outra. No quinto ano (Gráfico 5), a diferença no aprendizado em Matemática a favor dos meninos é muito pequena - varia de 1 ponto, em 2007, a 2,7, em 2011. Por isso, as linhas estão quase sobrepostas. Mas, no nono ano, as médias dos meninos superam as das meninas; a menor diferença foi observada em 2013, 5,1 pontos e a maior em 2009, 9,6 pontos.

A literatura educacional possui inúmeras evidências que corroboram os nossos achados, com múltiplas explicações 196 (Buchmann, Diprete e McDaniel, 2008). Uma hipótese, para o caso brasileiro, é a enorme seletividade escolar, devido às altas taxas de reprovação e abandono ou evasão, que afeta mais os meninos que as meninas (Alves e Ferrão, 2019; Alves e Soares, 2019). Os alunos que permanecem até o nono ano são mais selecionados do que as alunas, o que favorece o aprendizado desse grupo em uma área de conhecimento que, no processo de socialização, tanto na família como na escola, está mais associada a habilidades masculinas. 


\section{Gráfico 3}

Evolução do aprendizado em leitura no 50 ano do ensino fundamental por gênero: 2007 a 2017

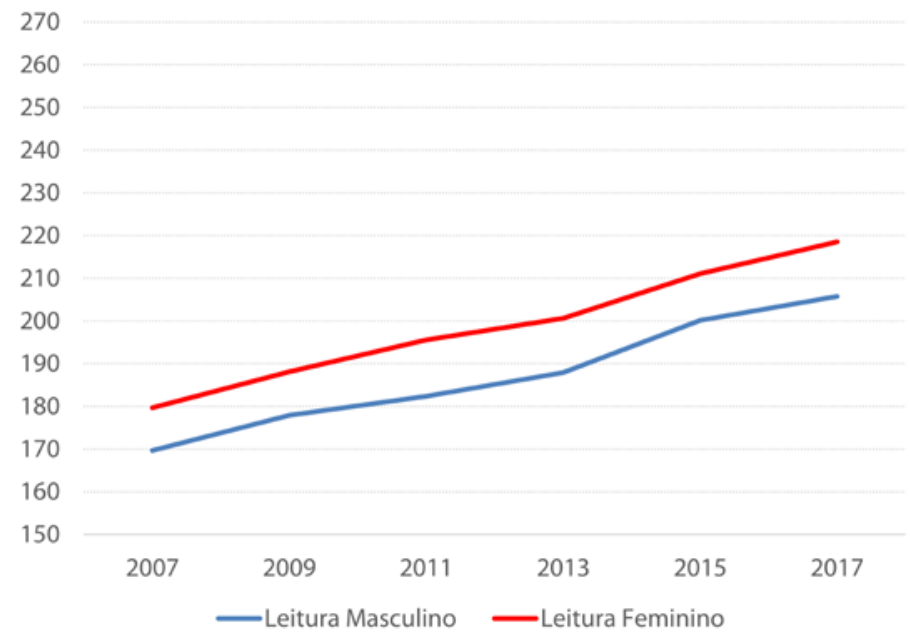

Fonte: Elaboração própria com dados da Prova Brasil/Inep.

\section{Gráfico 4}

Evolução do aprendizado em leitura no 9o ano do ensino fundamental por gênero: 2007 a 2017

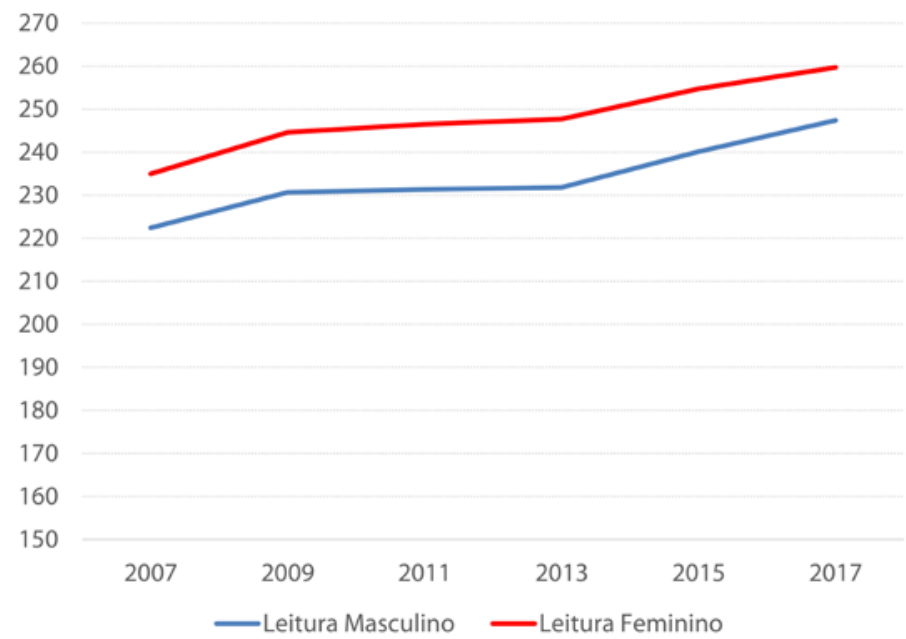

Fonte: Elaboração própria com dados da Prova Brasil/Inep. 


\section{Gráfico 5}

Evolução do aprendizado em matemática no 50 ano do ensino fundamental por gênero: 2007 a 2017

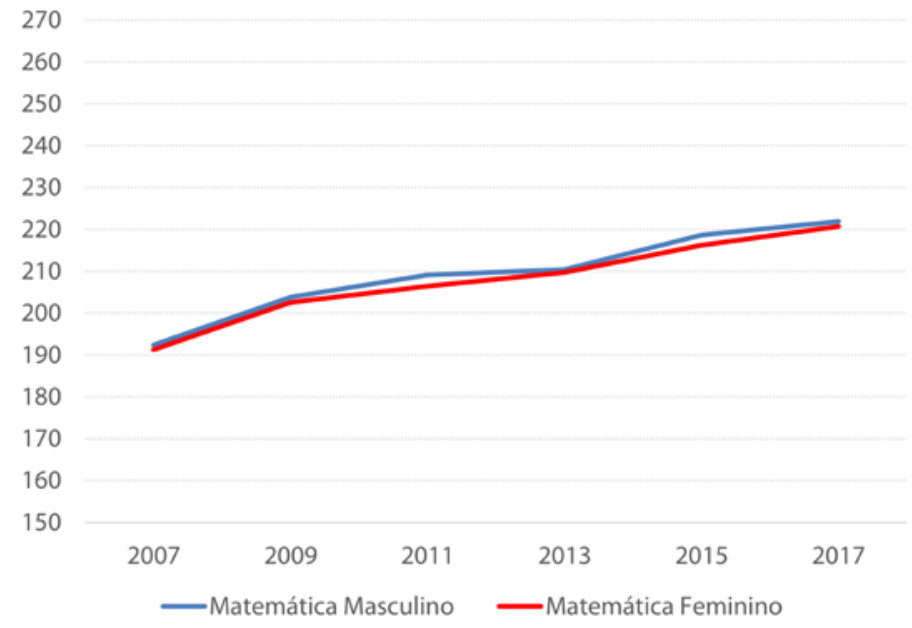

Fonte: Elaboração própria com dados da Prova Brasil/Inep.

Gráfico 6

Evolução do aprendizado em matemática no 90 ano do ensino fundamental por gênero: 2007 a 2017

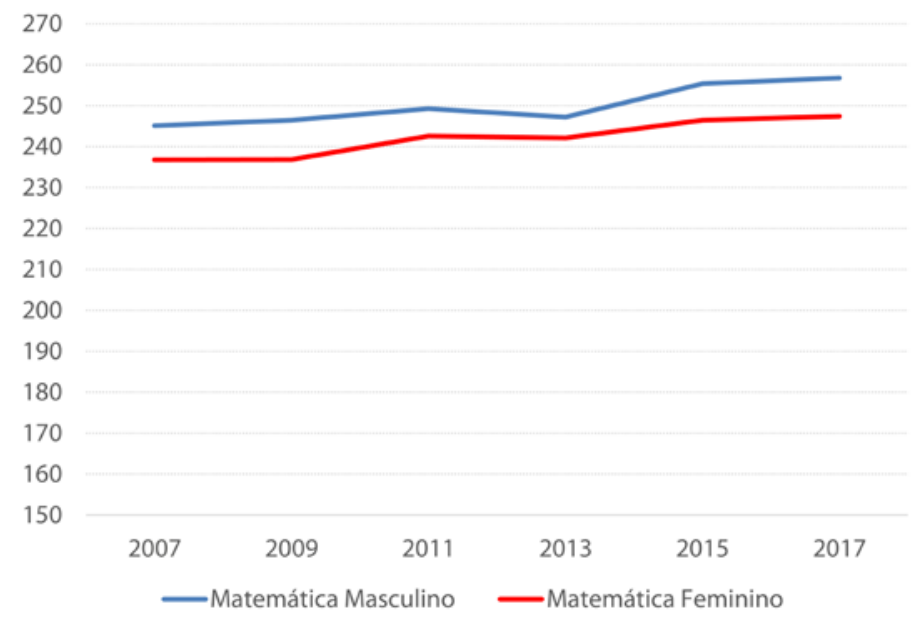

Fonte: Elaboração própria com dados da Prova Brasil/Inep. 
Os próximos resultados destacam o hiato de aprendizado somente no quinto ano em matemática. O Gráfico 7 apresenta o hiato por raça/cor. As diferenças são bem elevadas, há uma hierarquia nas médias dos alunos brancos, pretos e pardos, sendo que as diferenças entre brancos e pretos são enormes e crescentes. Em 2007, o hiato era de 16,7 pontos e, em 2017, cresceu para 27,5 pontos. Essa diferença equivale a mais de um ano de escolaridade na escala da Prova Brasil. Socialmente, é preocupante que esse padrão de desigualdade cresça em vez de diminuir, sendo que, em 2017, o desempenho dos alunos pretos declinou, ao contrário dos outros grupos.

O Gráfico 8 mostra o hiato por faixa do NSE dos alunos. Este foi calculado pela síntese de itens respondidos pelos alunos nos questionários contextuais nas avaliações educacionais da educação básica realizadas pelo Inep, empregando metodologia descrita por Alves, Soares e Xavier (2014). Para esta análise, a escala do NSE, originalmente contínua, foi seccionada em quintis. Notem que as linhas de todos os grupos têm inclinação ascendente, o que indica que estão melhorando o aprendizado em Matemática. Mas o gráfico mostra o maior hiato dentre todas as variáveis, as linhas estão mais próximas em 2007 e vão se afastando a cada edição a Prova Brasil. Isso significa que os alunos com NSE mais alto estão aprendendo muito mais do que aqueles com NSE mais baixo. Em 2007, a diferença entre o $1^{\circ}$ e o $5^{\circ}$ quintil (os $20 \%$ com NSE mais alto e os $20 \%$ com NSE mais baixo) era de 20,9 pontos. Em 2017, a diferença chegou a 38,3 pontos. O ápice foi em 2013, quando essa diferença atingiu 42 pontos, que equivale a dois anos de escolaridade. É como se o aprendizado do aluno com NSE mais baixo fosse compatível com o de um aluno do terceiro ano. 
Gráfico 7

Evolução do aprendizado em matemática no 50 ano do ensino fundamental por raça/cor: 2007 a 2017

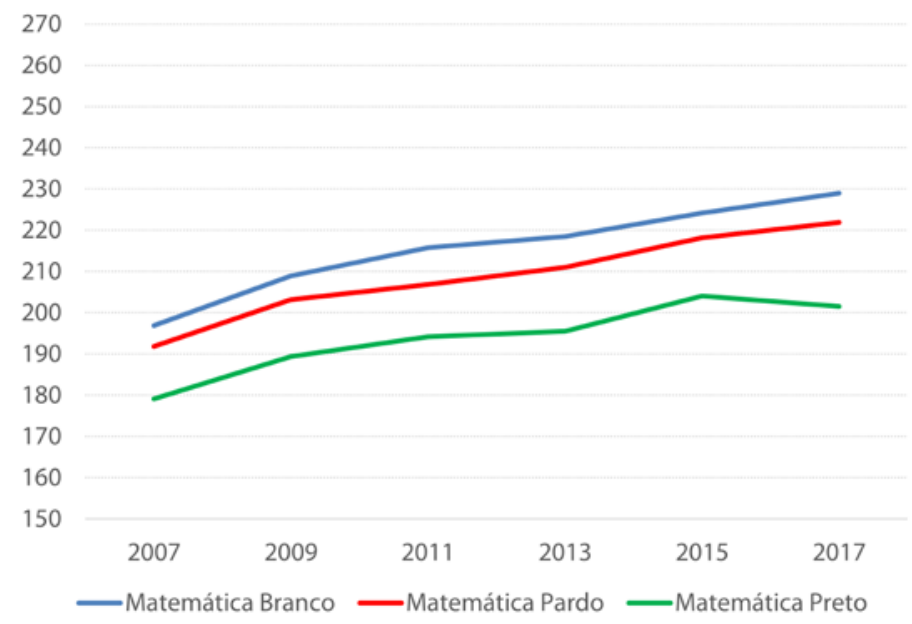

Fonte: Elaboração própria com dados da Prova Brasil/Inep.

Gráfico 8

Evolução do aprendizado em matemática no 50 ano do ensino fundamental por NSE: 2007 a 2017

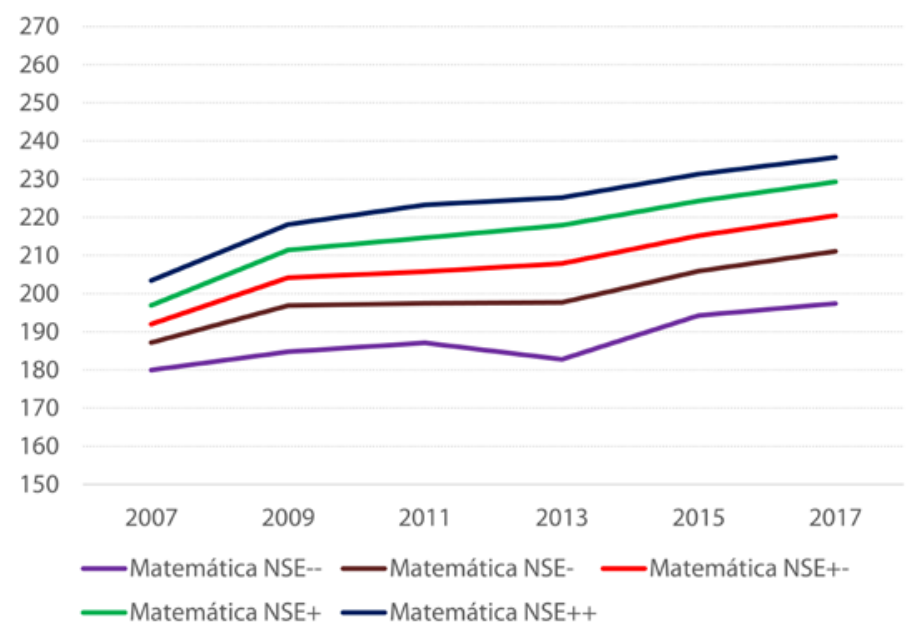

Fonte: Elaboração própria com dados da Prova Brasil/Inep. 
Até este ponto, analisamos as trajetórias das médias de aprendizado por grupo de origem social. Quando todas essas variáveis são examinadas simultaneamente, as diferenças ficam muito mais acirradas. Para ilustrar essa questão mais complexa, estudamos o hiato por grupos definidos por múltiplas características. Agrupamos três variáveis e algumas categorias: sexo (masculino e feminino); cor (branco e preto); NSE ( $1^{\circ}$ quintil e $5^{\underline{o}}$ quintil). No Gráfico 9 estão os resultados dos alunos definidos por cor e sexo entre aqueles que estão no $1^{\circ}$ quintil da variável NSE (mais baixo). No Gráfico 10 estão os mesmos grupos de cor e sexo, variando o NSE que corresponde ao quintil mais alto. Os dois gráficos estão na mesma escala.

O que chama atenção é que, ao variar o NSE do mais baixo para o mais alto, os alunos brancos (meninas ou meninos) se destacam. Se o exercício é feito para o lado contrário, os grupos se confundem. Isso indica que, na situação de mais pobreza, há um círculo vicioso no qual os resultados educacionais são ruins para todos os grupos sem distinção. Quando o cenário socioeconômico melhora, observa-se um círculo virtuoso favorável aos alunos brancos (meninos ou meninas), que apresentam resultados muito superiores aos dos alunos pretos na mesma condição socioeconômica. Ou seja, para os alunos pretos não basta melhorar a condição de vida para que o desempenho escolar seja equiparado ao do aluno branco. Este resultado corrobora achados anteriores sobre a dificuldade de as escolas equalizarem diferenças raciais sem políticas para o enfrentamento dessas desigualdades (Soares e Alves, 2003). 


\section{Gráfico 9}

Evolução do aprendizado em Matemática no 50 ano do ensino fundamental por grupos múltiplos: 2007 a 2017 (1ำ quintil)

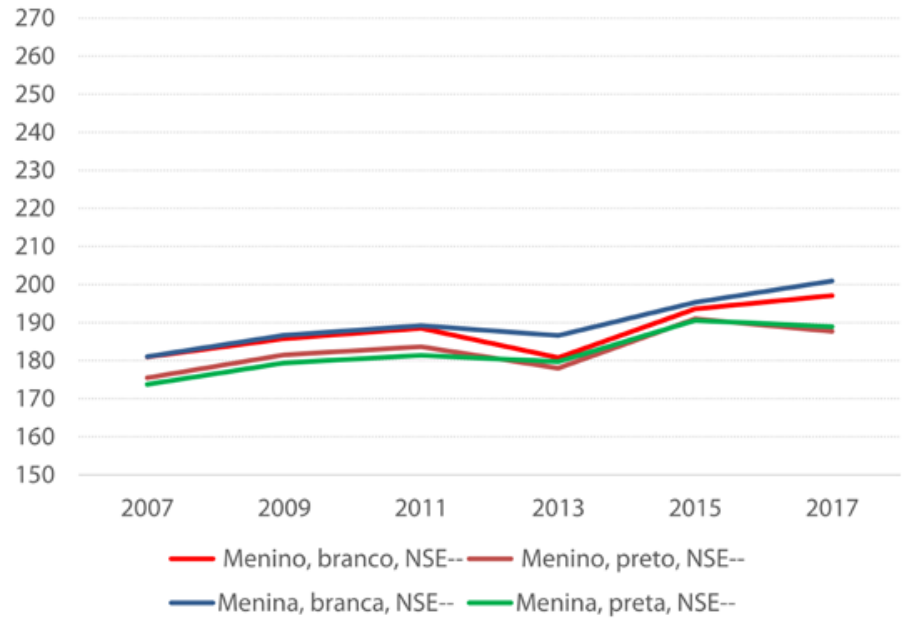

Fonte: Elaboração própria com dados da Prova Brasil/Inep.

\section{Gráfico 10}

Evolução do aprendizado em Matemática no 50 ano do ensino fundamental por grupos múltiplos: 2007 a 2017 (5ำ quintil)

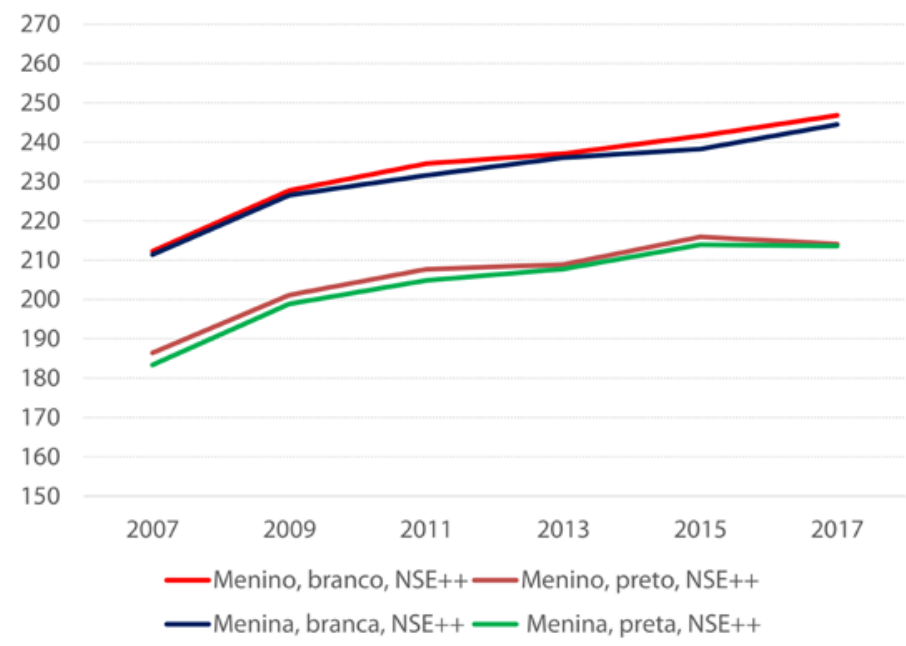

Fonte: Elaboração própria com dados da Prova Brasil/Inep. 


\section{Efeito escola e exclusão intraescolar: desigualdades de oportunidades educacionais nas escolas públicas de ensino fundamental}

Em "Os excluídos do interior" os sociólogos Bourdieu e Champagne (2001) argumentam que um dos efeitos paradoxais da democratização da educação é a desvalorização dos níveis escolares à medida que a expansão dava acesso a grupos sociais que antes estavam excluídos. Referindo-se à França, os autores argumentam que "não bastava ter acesso ao ensino secundário para ter êxito nele, ou ter êxito no ensino secundário para ter acesso às posições sociais que podiam ser alcançadas com os certificados escolares" (Bourdieu; Champagne, 2001, p. 220).

No Brasil, a expansão ainda não conseguiu universalizar nem mesmo toda a educação básica, que compreende a educação infantil, o ensino fundamental e médio. Apenas o ensino fundamental está próximo à universalização, com $98 \%$ das crianças e jovens dos 6 aos 14 anos matriculadas. Isso não significa que esses alunos estão em adequação idade-série, uma vez que $24 \%$ dos jovens de 16 anos não concluíram o ensino fundamental porque têm trajetória escolar com intercorrências (reprovação e/ou abandono), e muitas não aprendem o que se espera em cada etapa escolar. ${ }^{1}$

Para descrever esse problema no Brasil, Soares e colaboradores utilizaram o termo exclusão intraescolar, que caracteriza a situação do aluno matriculado em uma escola, mas que não aprende as competências em leitura e Matemática de maneira compatível com a etapa de ensino em que se encontra (Unesco, 2012). Os autores descreveram o aprendizado segundo níveis, em vez de médias, atribuindo um sentido normativo à escala de proficiência, a saber:

\footnotetext{
1 Os dados são produzidos pelo IBGE e Inep, mas os indicadores educacionais de rendimento são mais facilmente consultados no site do Observatório do PNE (http://www.observatoriodopne.org.br) e os indicadores de aprendizado no site do QEdu (http://www.observatoriodopne.org.br).
} 
nível abaixo do básico, nível básico, nível proficiente e nível adequado. Os alunos no nível abaixo do básico são aqueles em situação de exclusão escolar.

Essa interpretação da escala de proficiência segundo níveis foi aplicada aos dados da Prova Brasil (Alves e Xavier, 2016; Soares, Alves e Xavier, 2016; Unesco, 2017) para analisar o efeito dos estabelecimentos de ensino e a relação deste com indicadores escolares de eficácia. A abordagem metodológica consistiu: (1) na estimação dos efeitos das escolas brasileiras; e (2) na análise descritiva da relação desses efeitos com fatores associados às escolas.

O efeito escola é "o efeito característico de determinado estabelecimento de ensino, o 'valor agregado' pelo estabelecimento frequentado" (Bressoux, 2011, p. 257). É um medida do quanto a escola é capaz de elevar o nível médio dos alunos, levando-se em conta suas diferença de origem social ou aquelas anteriores ao ingresso na 204 escola. Nesse estudo, adaptamos essa noção para estimar o efeito das escolas em relação aos níveis de aprendizado (Soares, Alves e Xavier, 2016), obtendo dois tipos de efeito das escolas: efeito 1, que consiste na capacidade da escola em diminuir as chances de seus alunos estarem em situação de exclusão (nível abaixo do básico); e efeito 2, que é a capacidade da escola em aumentar as chances de seus alunos estarem em situação de adequação (nível adequado/avançado).

Os efeitos das escolas foram estimados por meio do ajuste de modelos de regressão hierárquica multinomial, nos quais a variável resposta, a proficiência dos alunos, foi seccionada em três classes: abaixo do básico, básico (categoria de referência) e adequado (soma do proficiente e avançado). Como variáveis de controle, foram incluídas aquelas relacionadas às características de origem social e trajetória escolar do aluno (sexo, atraso escolar, cor/raça, NSE do aluno e série escolar) e uma variável de controle contextual da escola (NSE médio da escola). 
Os fatores escolares foram obtidos por meio da síntese de itens dos questionários contextuais da Prova Brasil respondidos pelo diretor e pelos professores, além de um instrumento sobre as escolas. Mais detalhes podem ser consultados em Unesco (2017).

A análise mostrou que a maioria dos fatores escolares tem associação linear com os desfechos de interesse deste estudo, isto é, a redução das chances de exclusão escolar e o aumento das chances de adequação do aprendizado. Como exemplos dos resultados obtidos, descrevemos a relação entre os dois tipos de efeitos das escolas com os fatores: liderança administrativa, gestão participativa, coesão da equipe pedagógica e equipamentos da escola.

$\mathrm{O}$ fator liderança administrava reúne itens que mensuram a capacidade do diretor de garantir recursos financeiros para o funcionamento e a manutenção da escola, assim como a atenção em relação aos problemas administrativos. A gestão participativa se refere às instâncias e processos participativos que envolvem a direção, os professores, os alunos e a comunidade externa à escola. A coesão da equipe pedagógica é resultado da síntese de itens sobre o modo de trabalho e a convivência entre diretores e demais membros da equipe pedagógica, como a troca de ideias e compartilhamento de ações pedagógica. $\mathrm{O}$ fator equipamento reúne informações sobre a existência na escola de computadores, internet, aparelhos de audiovisual e outros para fins pedagógicos.

A associação desses fatores com as medidas de efeito escola tipo 1 e tipo 2, e por área de conhecimento, estão nos gráficos 11 a 14. Os fatores, originalmente em escala de desvios-padrão, foram seccionados em quartis. Observamos nos gráficos as médias dos efeitos tipo 1 e tipo 2 segundo os quartis dos fatores. Notem que a associação é sempre positiva nos quatro gráficos.

Por exemplo, no Gráfico 11, observamos as médias do efeito tipo 1 (barra azul) e efeito tipo 2 (barra vermelha) 
por quartil do fator liderança administrativa para leitura (metade esquerda do gráfico) e Matemática (metade direita). Observem que as barras, tanto para o efeito tipo 1, quanto para o tipo 2, estão com valores negativos nos dois primeiros quartis. Nos quartis 3 e 4, as barras apresentam valores positivos, sendo o maior valor exatamente do quartil mais alto. Isso significa que nas escolas em que a liderança administrativa é mais eficaz, os alunos têm menos chances de estar em situação de exclusão escolar (efeito tipo 1) e mais chances de ter aprendizado adequado (efeito tipo 2).

No fator gestão participativa (Gráfico 12), apenas no primeiro quartil os efeitos têm escores negativos. Ou seja, um pequeno aumento no nível de participação dos sujeitos escolares está associado à redução da exclusão intraescolar (efeito tipo 1) e aumento nas chances de aprendizado adequado (efeito tipo 2). O mesmo padrão linear pode ser observado nos outros dois gráficos.

206 De forma geral, os resultados oferecem evidências de que as condições da oferta educativa - um direito que deveria estar garantido igualmente para todos - potencializam as chances de os alunos terem os resultados educacionais desejados nas escolas. Vale registrar que essas associações foram descritas a partir de resultados obtidos em modelos estatísticos multivariados, que incluiu controle de caraterísticas do aluno e da escola. Se esse controle não fosse feito, as associações seriam muito mais fortes. 
Gráfico 11

Relação entre os efeitos tipos 1 e 2 com o fator liderança administrativa

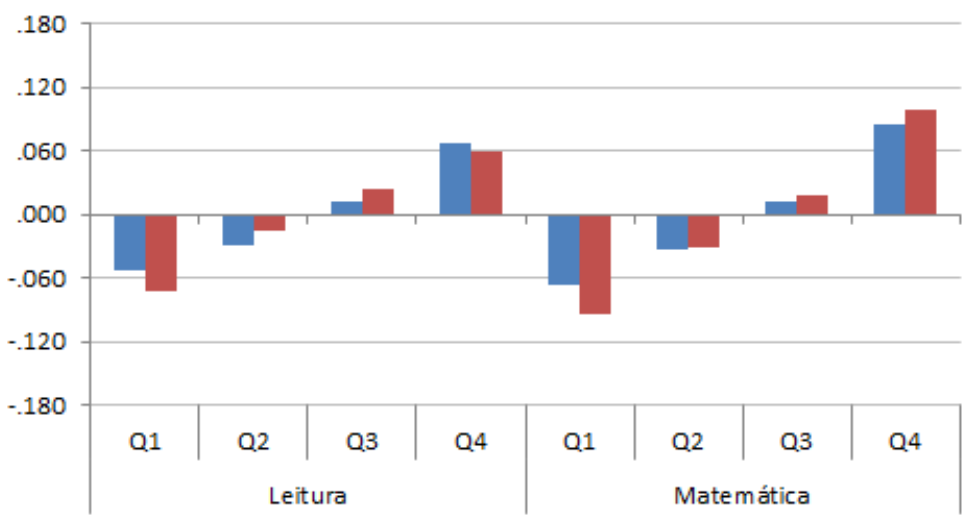

Efeito 1 Efeito 2

Gráfico 12

Relação entre os efeitos tipos 1 e 2 com o fator gestão participativa

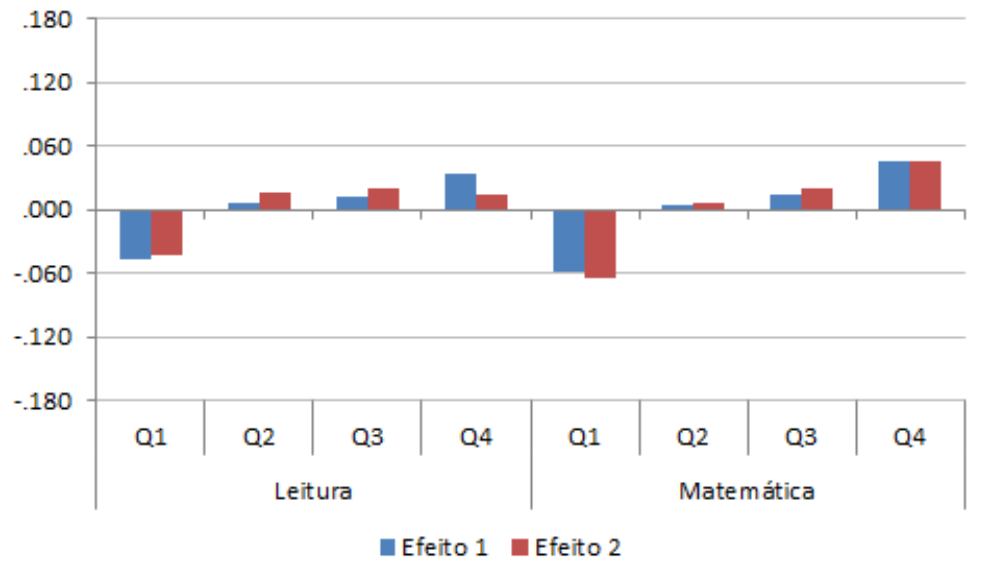


Gráfico 13

Relação entre os efeitos tipos 1 e 2 com o fator coesão da equipe pedagógica

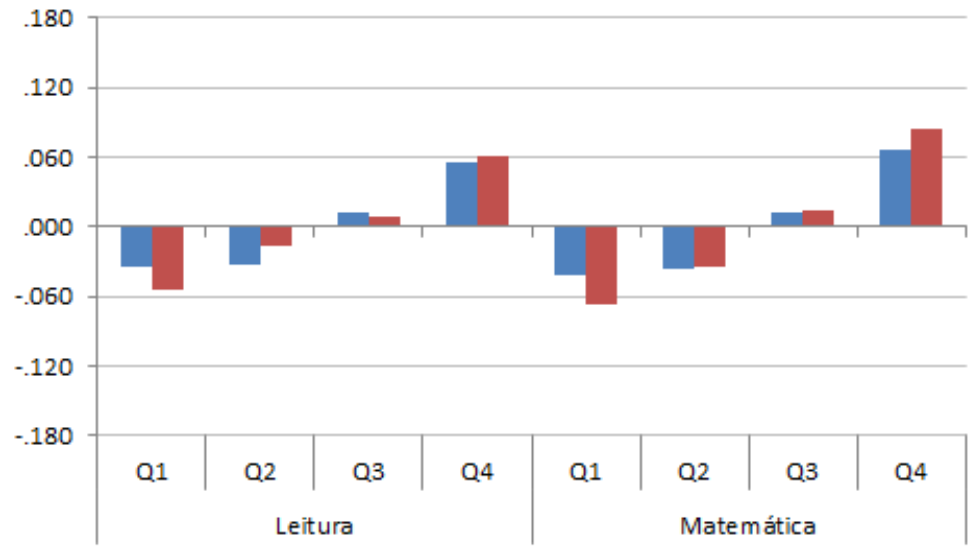

Efeito 1 Efeito 2

Gráfico 14

Relação entre os efeitos tipos 1 e 2 com o fator equipamentos

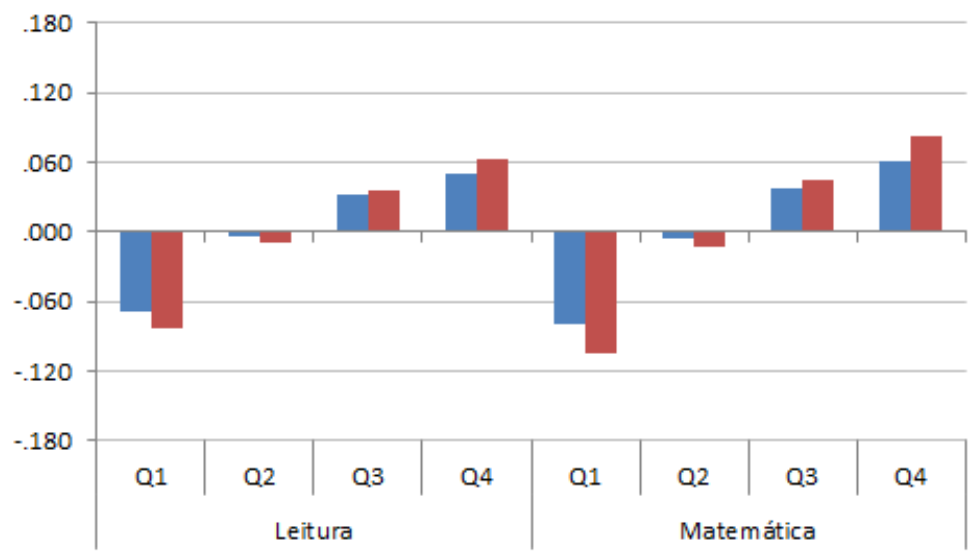

Efeito 1 Efeito 2

Fonte: Unesco (2017). 


\section{Considerações finais}

Os estudos com dados quantitativos aumentaram substancialmente nosso conhecimento sobre os mecanismos estruturais que caracterizam as desigualdades educacionais e contribuíram para o fortalecimento da sociologia da educação. O conhecimento produzido nesse campo pode contribuir para mudar ou manter as formas de escolarização em cada tempo e lugar. Nas palavras de Bourdieu (1982, p. 35), “o que deve ser feito quando se enuncia uma lei social anteriormente ignorada [?] [...] ela pode se tornar um objeto de luta: luta para conservar, conservando as condições de funcionamento da lei; luta para transformar, modificando estas condições". O paradigma da reprodução, do qual esse autor é uma das principais referências, não poderia ter sido demonstrado empiricamente sem as estatísticas da demografia educacional.

A sociologia da educação tem grande potencial para informar as políticas educacionais, que são fundamentais para reduzir diferenças educacionais. As cotas nas universidades, o Fundo de Desenvolvimento da Educação Básica (Fundeb) criado para reduzir as desigualdades entre sistemas de ensino, as definições curriculares e a noção de "conhecimento poderoso”, introduzida por Young (2007) como ferramenta para redução das desigualdades, são exemplos de políticas públicas informadas pela sociologia da educação.

Apesar desses avanços, as teorias do campo têm tido menos sucesso em oferecer explicações que orientem ações para reduzir as desigualdades no nível das escolas. Nossos achados sugerem que as desigualdades não serão vencidas sem intervenções pedagógicas das escolas, sem que elas ampliem as oportunidades educacionais dos alunos em desvantagem com melhorias das condições de escolarização.

As evidências empíricas, com os dados das avaliações educacionais, indicam algumas pistas sobre isso. Os resultados 
permitem fazer uma avaliação mais cuidadosa de práticas e situações que possibilitam às escolas melhorarem a capacidade de produzir efeitos desejáveis de promoção da inclusão educacional. Em nosso programa de pesquisas, desenvolvemos novos estudos com dados do Inep, caracterizamos a infraestrutura das escolas públicas, descrevemos a complexidade desse insumo e a sua relação com diferenças regionais, locais e resultados educacionais (Alves, Soares, 2013; Alves, Xavier, 2018; Alves, Xavier, Cunha, 2019; Alves, Xavier, Paula, 2019; Soares, Alves, Xavier, 2016; Unesco, 2019).

Reconhecemos que resultados obtidos em estudos que não são experimentais devem ser interpretados com parcimônia. Um caminho a ser trilhado sugere aprofundar o conhecimento necessário para intervenções, realizar pesquisas interdisciplinares que lidam com as múltiplas dimensões da desigualdade. Também podem ser interessantes as pesquisas com métodos mistos, cujos achados podem 210 produzir conhecimento generalizável e, ao mesmo tempo, uma visão mais profunda sobre os mecanismos sociais sutis e muitas vezes invisíveis que moldam experiências vividas no contexto escolar.

\section{Agradecimentos}

Este artigo foi desenvolvido no âmbito do projeto interinstitucional "Estratificação da educação básica brasileira: uma abordagem multidimensional”, apoiado pelo Conselho Nacional de Desenvolvimento Científico e Tecnológico (CNPq) (processo 440172/2017-9) para o "I Seminário Ciências Socias e Educação", realizado na Universidade Estadual do Rio de Janeiro (Uerj), em 23 e 24 de maio de 2019.

\section{Maria Teresa Gonzaga Alves}

é professora associada do Departamento de Ciências Aplicadas à Educação e do Programa de Pós-Graduação em Educação da Universidade Federal de Minas Gerais (UFMG), 
colaboradora do Centro de Matemática Aplicada à Previsão e Decisão Econômica (Cemapre) da Universidade de Lisboa, Portugal, e líder do Núcleo de Pesquisa em Desigualdades Escolares (CNPq).

\section{Bibliografia}

ALVES, Maria Teresa Gonzaga; FERRÃO, Maria Eugênia. 2019.

Uma década da Prova Brasil: evolução do desempenho e da aprovação. Estudos em Avaliação Educacional, v. 30, n. 75, p. 688-720.

ALVES, Maria Teresa Gonzaga; FRANCO, Creso. 2008. A pesquisa em eficácia escolar no Brasil. In: BROOKE, Nigel; SOARES, José Francisco (ed.). Pesquisa em eficácia escolar: origem e trajetórias. Belo Horizonte: Ed. UFMG. p. 482-500.

ALVES, Maria Teresa Gonzaga; SOARES, José Francisco. 2007. As pesquisas sobre o efeito das escolas: contribuições metodológicas para a sociologia da educação. Sociedade e Estado, v. 22, n. 2, p. 435-473.

ALVES, Maria Teresa Gonzaga; SOARES, José Francisco. 2013. Contexto escolar e indicadores educacionais: condições desiguais para a efetivação de uma política de avaliação educacional. Educação $e$ Pesquisa, v. 39, n. 1, p. 177-194. doi: 10.1590/S1517-97022013000100012 ALVES, Maria Teresa Gonzaga; SOARES, José Francisco. 2019. Trajetórias escolares no ensino fundamental: análise longitudinal do Censo Escolar. Paper apresentado no X Encontro da Associação Brasileira de Avaliação Educacional (Abave), São Paulo, 28 a 30 de agosto.

ALVES, Maria Teresa Gonzaga; SOARES, José Francisco; XAVIER, Flavia Pereira. 2014. Índice socioeconômico das escolas de educação básica brasileiras. Ensaio: Avaliação e Políticas Públicas em Educação, v. 22, n. 84. p. 671-703.

ALVES, Maria Teresa Gonzaga; SOARES, José Francisco; XAVIER, Flavia Pereira. 2016. Desigualdades Educacionais no Ensino Fundamental de 2005 a 2013: hiato entre grupos sociais. Revista Brasileira de Sociologia, v. 4 , p. $49-81$.

ALVES, Maria Teresa Gonzaga; XAVIER, Flavia Pereira. 2016. Construção de indicadores para descrever desigualdades de aprendizado na Prova Brasil. Estudos em Avaliação Educacional, v. 27, p. 782-815.

ALVES, Maria Teresa Gonzaga; XAVIER, Flavia Pereira. 2018. Indicadores multidimensionais para avaliação da infraestrutura escolar: o ensino fundamental. Cadernos de Pesquisa, São Paulo, v. 48, n. 169, p. 708-746. 
ALVES, Maria Teresa Gonzaga; XAVIER, Flavia Pereira; CUNHA, Maria Amália de Almeida. 2019. Desigualdades escolares: concepção teórica e indicadores. Paper apresentado no I Seminário do Projeto Estratificação da Educação Básica Brasileira: uma abordagem multidimensional (CNPq) IESP-UERJ, Rio de Janeiro, 5 e 6 de setembro.

ALVES, Maria Teresa Gonzaga; XAVIER, Flavia Pereira; PAULA, Túlio Silva de. 2019. Modelo conceitual para avaliação da infraestrutura escolar no ensino fundamental. Revista Brasileira de Estudos Pedagógicos, v. 100, n. 297, p. 297-330.

BOURDIEU, Pierre. 1982. Questões de sociologia. Rio de Janeiro: Marco Zero. BOURDIEU, Pierre; CHAMPAGNE, Patrick. 2001. Os excluídos do interior. In: NOGUEIRA, Maria Alice; CATANI, Afrânio (org.). Escritos de educação. 3. ed. Petrópolis: Vozes. p. 217-227.

BRESSOUX, Pascal. 2011. Efeito estabelecimento. In: VAN ZANTEN, Agnes (coord.). Dicionário de educação. Petrópolis: Vozes. p. 275-279.

BROOKE, Nigel; SOARES, José Francisco. 2008. Comentários da Seção 1. In: BROOKE, Nigel; SOARES, José Francisco (ed.). Pesquisa em eficácia escolar: origem e trajetórias. Belo Horizonte: Ed. UFMG, p, 14-22.

BROOKOVER, Wilbur B. et al. 1979. Schools, social systems and student achievement: schools can make a difference. New York: Praeger.

BUCHMANN, Claudia; DIPRETE, Thomas A.; MCDANIEL, Anne. 2008. Gender inequalities in education. Annual Review of Sociology, v. 34, n. 1, p. 319-337.

CAVALCANTI, Cacilda Rodrigues; OLIVEIRA, Rosimar de Fátima. 2019. Mecanismos redistributivos na assistência financeira da união no âmbito da educação básica. Educação em Revista, v. 35, p. e194676.

COLEMAN, James S. et al. 1966. Equality of educational opportunity. Washington, DC: US Government Printing Office.

FERRARO, Alceu Ravanello. 2002. Analfabetismo e níveis de letramento no Brasil: o que dizem os censos? Educação Ẽ Sociedade, v. 23, n. 81, p. 21-47. FORQUIN, Jean-Claude. 1995. A Sociologia das desigualdades de acesso à educação: principais orientações, principais resultados desde 1965 . In. FORQUIN, Jean-Claude. (ed.). Sociologia da educação: dez anos de pesquisas. Petrópolis: Vozes. p. 19-78.

GOLDSTEIN, Harvey. 2001. Modelos de realidade: novas abordagens para a compreensão de processos educacionais. In: FRANCO, Creso (ed.). Avaliação, ciclos e promoção na educação. Porto Alegre: Artmed. p. 84-99. HAMBLETON, Ronald K. 1993. Principles and selected applications of Item Response Theory. In: LINN, Robert L. (ed.). Educational 
measurement. 3. ed. Washington, DC: American Council on Education and the National Council on Measurement in Education. p. 147-200.

KARABEL, Jerome; HALSEY, A. H. 1977. Introduction. In: KARABEL, Jerome; HALSEY, A. H. Power and ideology in education. New York: Oxford University Press. p. 1-85.

KLEIN, Ruben. 2003. Produção e utilização de indicadores educacionais: metodologia de cálculo de indicadores do fluxo escolar da educação básica. Revista Brasileira de Estudos Pedagógicos, v. 84, n. 206/207/208, p. 107-157.

LEE, Valerie E. 2008. Utilização e modelos hierárquicos lineares para estudar contextos sociais: o caso dos efeitos da escola. In: BROOKE, Nigel; SOARES, José Francisco (ed.). Pesquisa em eficácia escolar: origem e trajetórias. Belo Horizonte: Ed. UFMG. p. 273-296.

MAFRA, Leila de Alvarenga. 2003. A Sociologia dos estabelecimentos escolares: passado e presente de um campo de pesquisa em re-construção. In: ZAGO, Nadir; CARVALHO, Marília Pinto de; VILELA, Rita Amélia Teixeira (org.). Itinerários de pesquisa: perspectivas qualitativas de Sociologia da Educação. Rio de Janeiro: DP\&A. p. 109-136.

NOGUEIRA, Maria Alice. 1990. A sociologia da educação do final dos anos 60 / início dos anos 70: o nascimento do paradigma da reprodução. Em Aberto, v. 9, n. 46, p. 49-59.

RUTTER, Michael et al. 1979. Fifteen thousand hours: secondary schools and their effects on children. Somerset: Open Books.

SOARES, José Francisco. 2016. O direito à educação no contexto da avaliação educacional. Em Aberto, v. 29, p. 141-152.

SOARES; José Francisco; ALVES, Maria Teresa Gonzaga. 2003. Desigualdades raciais no sistema brasileiro de educação básica. Educação e Pesquisa, São Paulo, v. 29, n. 1, p. 147-165.

SOARES, José Francisco; ALVES, Maria Teresa Gonzaga; XAVIER, Flavia Pereira. 2016. Effects of brazilian schools on student learning. Assessment in Education: Principles, Policy and Practice, v. 23, n. 1, p. 75-97. doi: 10.1080/0969594X.2015.1043856

UNESCO. 2012. Exclusão intraescolar nas escolas públicas brasileiras: um estudo com dados da Prova Brasil 2005, 2007 e 2009. Brasília, DF: Unesco. (Série Debates ED, n. 4).

UNESCO. 2017. Desigualdades de aprendizado entre alunos das escolas públicas brasileiras: evidências da Prova Brasil (2007 a 2013). Brasília, DF: Unesco. (Série Debates ED, n. 5). Disponível em: https://bit.ly/3a1ZoDB. Acesso em: 5 ago. 2020. 
UNESCO. 2019. Qualidade da infraestrutura das escolas públicas de ensino fundamental brasileiras: indicadores com dados públicos e tendências de 2013, 2015 e 2017. Brasília, DF: Unesco. Disponível em: https:/ /bit.ly/3i9BAAx. Acesso em: 5 ago. 2020.

VALLE SILVA, Nelson do; HASENBALG, Carlos. 2000. Tendências de desigualdades educacional no Brasil. Dados, v. 43, n. 3, p. 423-445.

VALLE SILVA, Nelson do; SOUZA, Alberto Mello. 1986. Um modelo para análise da estratificação educacional no Brasil. Cadernos de Pesquisa, v. 58 , p. $40-57$.

WILLMS, J. Douglas. 1992. Monitoring school performance: a guide for educators. Washington, DC: Falmer.

YOUNG, Michael. 2007. Para que servem as escolas? Educação Ẽ Sociedade, v. 28, n. 101, p. 1287-1302. 


\section{CARACTERIZAÇÃO DAS DESIGUALDADES EDUCACIONAIS COM DADOS PÚBLICOS: DESAFIOS PARA CONCEITUAÇÃO E OPERACIONALIZAÇÃO EMPÍRICA}

\section{MARIA TERESA GONZAGA ALVES}

Resumo: Este artigo discute a importância dos dados públicos no campo da sociologia da educação. As desigualdades de acesso à educação formal, trajetórias e resultados educacionais por origem social, grupos raciais, gênero e condições das escolas, por exemplo, não poderiam ser medidas na população se não houvesse dados em larga escala produzidos por órgãos públicos. No Brasil, o Instituto Brasileiro de Geografia e Estatística (IBGE) e o Instituto Nacional de Estudos e Pesquisas Educacionais "Anísio Teixeira" (Inep) são agências estatais fundamentais para a produção de conhecimento científico em campo. São apresentados alguns resultados de pesquisas realizadas com dados do Inep: o primeiro descreve as desigualdades de resultados escolares das escolas públicas brasileiras, de acordo grupos sociais de 2007 a 2017; e o segundo mostra evidências sobre as oportunidades educacionais nas escolas públicas e a relação com os efeitos da escola.

Palavras-chave: Dados Públicos; Desigualdades Educacionais; Efeito Escola; Eficácia Escolar.

\section{CHARACTERIZATION OF EDUCATIONAL INEQUALITIES WITH PUBLIC DATA: CHALLENGES FOR CONCEPTUALIZATION AND EMIPIRICAL OPERATIONALIZATION}

Abstract: This paper discusses the importance of public data in the field of Sociology of Education. Inequalities in access to formal education, educational trajectories and outcomes by social background, racial groups, gender, and school conditions, for example, could not be measured in the population without large-scale data produced by 
public agencies. In Brazil, the Brazilian Institute of Geography and Statistics (IBGE) and the National Institute for Educational Studies and Research "Anisio Teixeira" (Inep) are key state agencies for the production of scientific knowledge in the field. Some findings of research conducted with data from Inep are presented: The first describes the inequalities of school outcomes of Brazilian public schools according to social groups from 2007 to 2017; the second shows evidence about the educational opportunities in public schools and the relationship with the school effects.

Keywords: Public Data; Educational Inequalities; School Effect; School Effectiveness.

Recebido: 18/09/2019 Aprovado: 05/08/2020 